\title{
PROPUESTA METODOLÓGICA DE GESTIÓN DE CUENCA PARA EL SANEMIENTO, EL CASO DE LA MICROCUENCA DE EL MUERTO
}

\author{
METHODOLOGICAL PROPOSAL OF WATERSHED \\ MANAGEMENT FOR SANITATION, EL MUERTO \\ WATERSHED CASE
}

\author{
Magaly Mendoza Guadarrama ${ }^{1}$ \\ Universidad Autónoma del Estado de México \\ Alejandro Rafael Alvarado Granados ${ }^{2}$ \\ Universidad. Toluca, México
}

http://dx.doi.org/10.15359/rgac.3-59.9

\section{RESUMEN}

El arroyo El Muerto, estado de México, presenta contaminación generada por las actividades humanas dentro de su microcuenca. La atención del problema de contaminación puede desarrollarse a través de las particularidades del manejo del líquido. Ante esta situación, se planteó el estudio del proceso de manejo del agua para comprenderlo a partir del análisis de cinco núcleos: biofísico, sociocultural, económico, tecnológico y político-administrativo, con la finalidad de encontrar una alternativa a las visiones simples del aprovechamiento del recurso y lograr una propuesta metodológica de gestión.

1 Ingeniero Civil y estudiante de posgrado de la Universidad Autónoma del Estado de México (UAEM). Facultad de Planeación Urbana y Regional, Calle Mariano Matamoros casi esquina Paseo Tollocan s/n, Colonia Universidad. Toluca, Estado de México, C.P. 50130. Correo electrónico: magly_sanz@hotmail.com

2 Doctor en Ciencias Ambientales por la UAEM. Facultad de Planeación Urbana y Regional, Calle Mariano Matamoros casi esquina Paseo Tollocan s/n, Colonia Universidad. Toluca, Estado de México, C.P. 50130. Correo electrónico: alex13_bum@hotmail.com 
Magaly Mendoza Guadarrama, Alejandro Rafael Alvarado Granados. Methodological proposal of watershed management for sanitation, El Muerto watershed case

Palabras clave: gestión integral de agua y cuenca, manejo, metodología, actores.

\begin{abstract}
El Muerto stream, in the State of Mexico, presents pollution generated by human activities within its micro-watershed. The management of the contamination problem can be developed through the characteristics of water management. Because of this situation, the study of the water management process was undertaken to understand it from a five-core analysis: biophysical, socio-cultural, economic, technological and political-administrative, in order to find an alternative to simple visions regarding water use, and achieve a methodological management proposal.
\end{abstract}

Keywords: integrated water and watershed management, handling, methodology, stakeholders.

\title{
Introducción
}

El agua es un elemento vital para la supervivencia de los ecosistemas y de cualquier sociedad humana; con sus características de elemento integrador, así como, de bien necesario para el desarrollo y la estabilidad económica. Esta imperiosa necesidad ha conducido a la apropiación del líquido hasta límites insostenibles mediante un modelo que Falkenmark (2003), Chávez y Chávez (2009) y Aboites (2009), denominaron aprovechamiento basado en la demanda.

En este sentido, la sobreexplotación y la mala calidad del agua pronostican, que los problemas relacionados con ella crecerán si las formas de su manejo no cambian. Es por ello, que el estudio de los temas relacionados con el manejo y gestión del vital líquido toma gran relevancia para las administraciones gubernamentales como para las sociedades en general.

La microcuenca del arroyo El Muerto (MM) manifiesta esta problemática por el proceso de eutrofización que padece el cuerpo de agua donde descarga el caudal del mencionado arroyo, que es contaminado por las actividades humanas en su cuenca de captación. Cabe destacar, que esta investigación es la continuación del proyecto titulado "Programa de Saneamiento de la Cuenca del Arroyo El Muerto" (Alvarado y otros, 2013a), del cual surgió una propuesta para el saneamiento de la MM a partir de distintas acciones, vislumbradas desde la descripción analítica y el diagnóstico sintético, que dio por resultado la presentación de cuatro proyectos que son: "Educación para el manejo del agua en la cuenca", "El Potrero", "Agro-ecosistemas" y "Tubos y plantas de tratamiento".

De estos cuatro proyectos, los resultados que aquí se presentan se encuentran en el denominado "Tubos y plantas de tratamiento", mismo que se pretende impulsar a partir de la tecnología desarrollada por Xochicalli 
A.C. ${ }^{3}$, la cual consiste en la instalación de una planta de tratamiento en la localidad de San Pedro Atlapulco, para el saneamiento de la microcuenca.

Lo anterior, no deja de lado que todos los proyectos son importantes y se complementan por estar interrelacionados, y forman la totalidad de la propuesta de manejo integral de la microcuenca. En este sentido, es importante conocer a los entes relacionados con la gestión del agua en la cuenca y, cuál es su postura ante la problemática y la tecnología propuesta para el saneamiento del arroyo, con el fin de implementar el proyecto de saneamiento y desarrollar la propuesta metodológica de gestión para mejorar las condiciones ecológicas y, además, contribuir a las actividades económicas y de habitación en la zona de estudio.

En ese sentido, este trabajo presenta la metodología de gestión, basada en el proceso de manejo del agua con el estudio del caso de MM, en el estado de México, cuyo ámbito rural y características sociopolíticas facilitan la generación de un proyecto de gestión social del agua; además, se presenta el resultado del análisis de los actores, el cuál define el rumbo y las pautas que hay que seguir para la implementación del proyecto.

\section{La microcuenca del arroyo EI Muerto}

La MM, se encuentra dentro de la subcuenca Almoloya-Otzolotepec, por lo que forma parte de la región hidrológica número 12 Lerma-Santiago y pertenece a la cuenca Lerma-Toluca y la subcuenca Almoloya Otzolotepec (INEGI, 2014). Se encuentra inscrita en la vertiente occidental de la sierra de Las Cruces, en la región conocida como el Valle de Toluca; tiene una extensión de 2121 ha y la ocupan parcialmente tres municipios del estado de México: Tianguistenco (2.2\%), Capulhuac (22.9\%) y Ocoyoacac $(75 \%)$, como se ilustra en la figura 1 .

3 La tecnología Xochicalli A.C. pretende la recuperación de los materiales contenidos en las aguas residuales, para su aprovechamiento, y así conseguir la depuración del agua, obtener los nutrientes y energía contenidos en las aguas residuales domésticas 
Figura 1. Cuenca del arroyo El Muerto

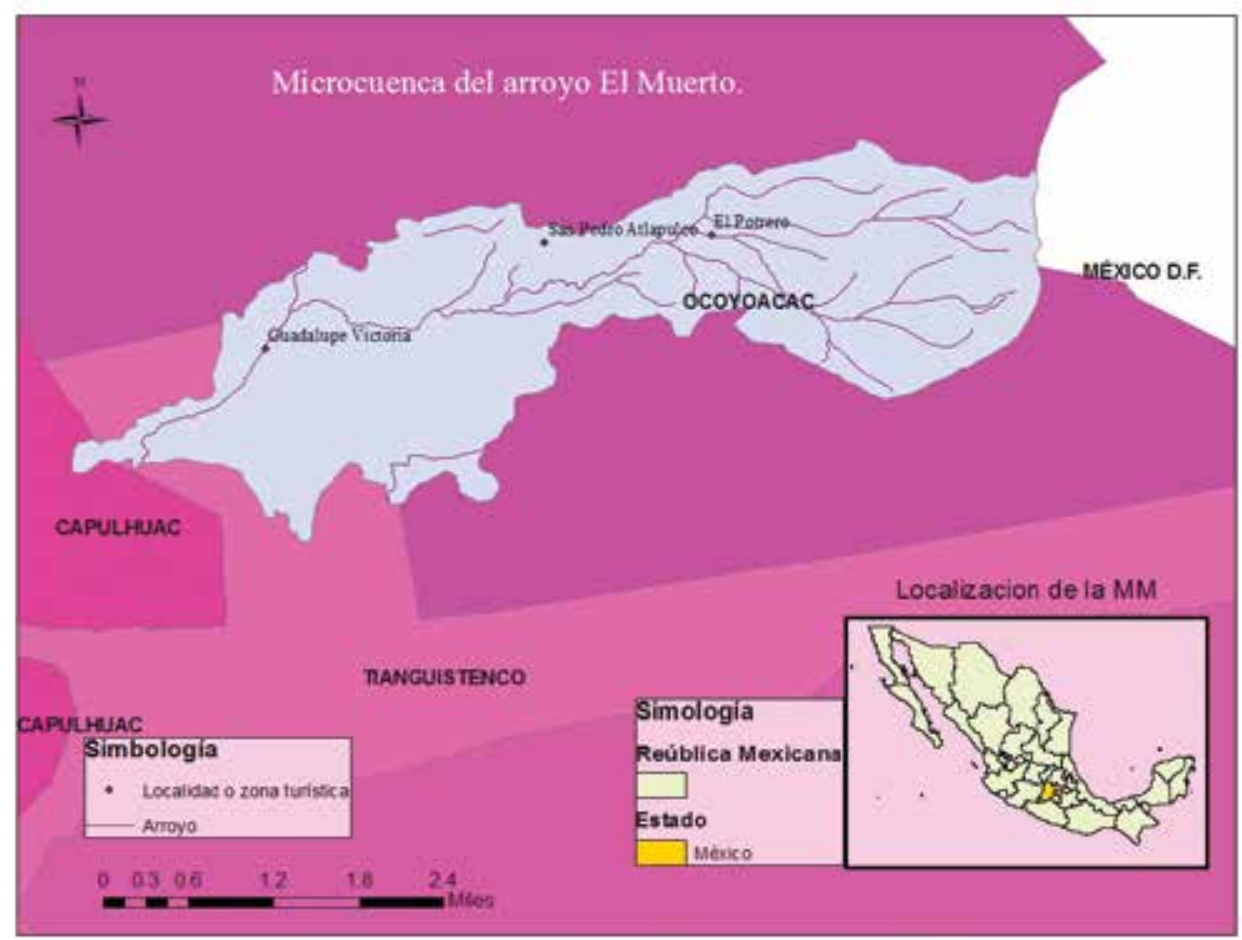

Fuente: Elaboración propia con base en INEGI, 2016.

\section{Una visión global para una problemática compleja}

Los fundamentos teórico-conceptuales del trabajo de investigación comprenden la Teoría General de los Sistemas y el pensamiento holístico, los cuales dan cuenta de la organización de los elementos de la cuenca incluidos para la gestión integral y los del proceso de manejo del agua como un sistema.

De esta manera, el proceso de manejo del agua es una herramienta cognitiva para comprender el funcionamiento de una cuenca en particular, con fines a la gestión de recursos hídricos, en el marco de la gestión integral de cuencas hidrológicas. Por lo anterior, se partió de la visión holística para explicar la plataforma sistémica en que se circunscribe la gestión de los recursos naturales dentro de la cuenca, a partir de la complejidad y su comprensión; el manejo y la gestión; la expresión espacial de la cuenca como zona de estudio y los actores que la conducen. 


\section{Sistemas y Complejidad}

Para comprender la complejidad de las relaciones hombre-naturaleza, así como las que involucran al hombre y su sociedad, se recurrió a los fundamentos teóricos propuestos por Von Bertalanffy (1976) y Savory (2005).

Ambos trabajos facilitaron la comprensión y definición de la cuenca como un sistema no sólo en el sentido hídrico, sino también en el social, económico, territorial y político, puesto que está conformada por un conjunto de elementos diversos, tales como población, viviendas, uso de suelo, orografía, precipitación, producción, consumo, expectativas, decisiones, etcétera, los cuales en conjunto interactúan con otros sistemas.

La comprensión y aplicación de estos fundamentos teóricos constituye una base para el conocimiento complejo, el cual se manifiesta de manera específica en la comprensión y explicación holística del funcionamiento espacial para la gestión integral de recursos naturales.

El uso de la Teoría General de los Sistemas (Von Bertalanffy, 1976) se aplica para comprender las actuaciones sociales que satisfacen sus necesidades hídricas a partir de la apropiación del recurso hídrico que es intervenido en algunas partes de su ciclo natural. Se precisa identificar las interdependencias entre los elementos de tres sistemas que comparten un mismo territorio: el proceso de manejo del agua, la gestión integral de cuenca y la cuenca hidrológica; los tres como sistemas complejos interdependientes e interrelacionados.

Este enfoque integrador permite incluir diversas dimensiones de análisis para entender las pertinencias, interrelaciones, causalidades y ponderaciones de los constituyentes de la gestión de la cuenca hidrológica. Basado en esta idea, las dimensiones que permiten dar cuenta del manejo y de la gestión del agua son: económica, tecnológica, biofísica, sociocultural y política-administrativa, como lo plantean Vega y otros (2008), Freitas (2006) y Alvarado y otros (2013a).

En el caso de la gestión integral de cuenca, los diferentes actores y sus actividades inciden de manera importante en el proceso de manejo, de ahí que la cuenca se considere como un sistema abierto con fenómenos naturales y sociales particulares. Así mismo, el manejo del agua da la pauta para los lineamientos de gestión, los cuales dirigirán el proyecto planteado para el saneamiento. 
El enfoque holístico propuesto por Allan Savory (2005) asevera que el deterioro ambiental se relaciona con el modo en que son tomadas las decisiones en el manejo de los recursos naturales. Es decir, que la actividad humana genera impactos positivos y negativos de gran envergadura o de poca extensión, pero siempre como consecuencia de su intervención en los procesos naturales de los ecosistemas.

El manejo holístico como herramienta cognitiva intenta integrar los procesos y objetos de diversa índole en una unidad que le denomina "un todo", para asegurar la sustentabilidad de las decisiones, y que de este modo se ejecuten proyectos con viabilidad y éxito.

El enfoque holístico, como base para la gestión integral, fomenta la visión de la realidad como un universo formado por unidades de diversos elementos, con características y formas propias, los cuales se relacionan intrínsecamente. Además, reconoce que el entorno está formado por patrones (de materia, energía y vida) que también participan en el todo, que no es transparente al análisis.

De esta manera, el enfoque holístico está fuertemente ligado a la toma de decisiones, pues representa una herramienta para comprender y entrever las posibles relaciones causales de un suceso. En ese orden de ideas, la gestión integral de cuencas hidrológicas queda seccionada, para fines de análisis, por los aspectos biofísicos, económicos, políticos, sociales, tecnológicos y ambientales; es decir, distintas manifestaciones de una realidad específica que la definen en conjuntaaaaao, así como a su entorno circundante.

\section{Manejo y gestión de cuenca}

El manejo del agua es el proceso social que incluye su apropiación, uso, aprovechamiento y desecho, con el objetivo de satisfacer diferentes necesidades hídricas. De acuerdo con Dourojeanni y otros (2002), el concepto de "manejo", en el ámbito de las cuencas hidrológicas, proviene de las escuelas forestales de Estados Unidos de Norteamérica por medio de la castellanización del término "management", en el sentido de manejar o manipular los aportes de agua con el fin de regular la escorrentía en calidad, cantidad y oportunidad.

Una forma en que los elementos y actividades realizadas en la cuenca están interrelacionadas, es a través del proceso de manejo del agua (Alvarado, González y Díaz, 2013), el cual implica las tres fases:

Revista Geográfica de América Central No 59 ISSN 1011-484X, julio-diciembre 2017, pp. 275-307 
apropiación-distribución, uso o aprovechamiento y descarga, que son intervenidas mediante acciones de gestión hídrica y realizadas a partir del proceso natural del ciclo hidrológico (figura 2)

Figura 2. Fases del manejo del agua

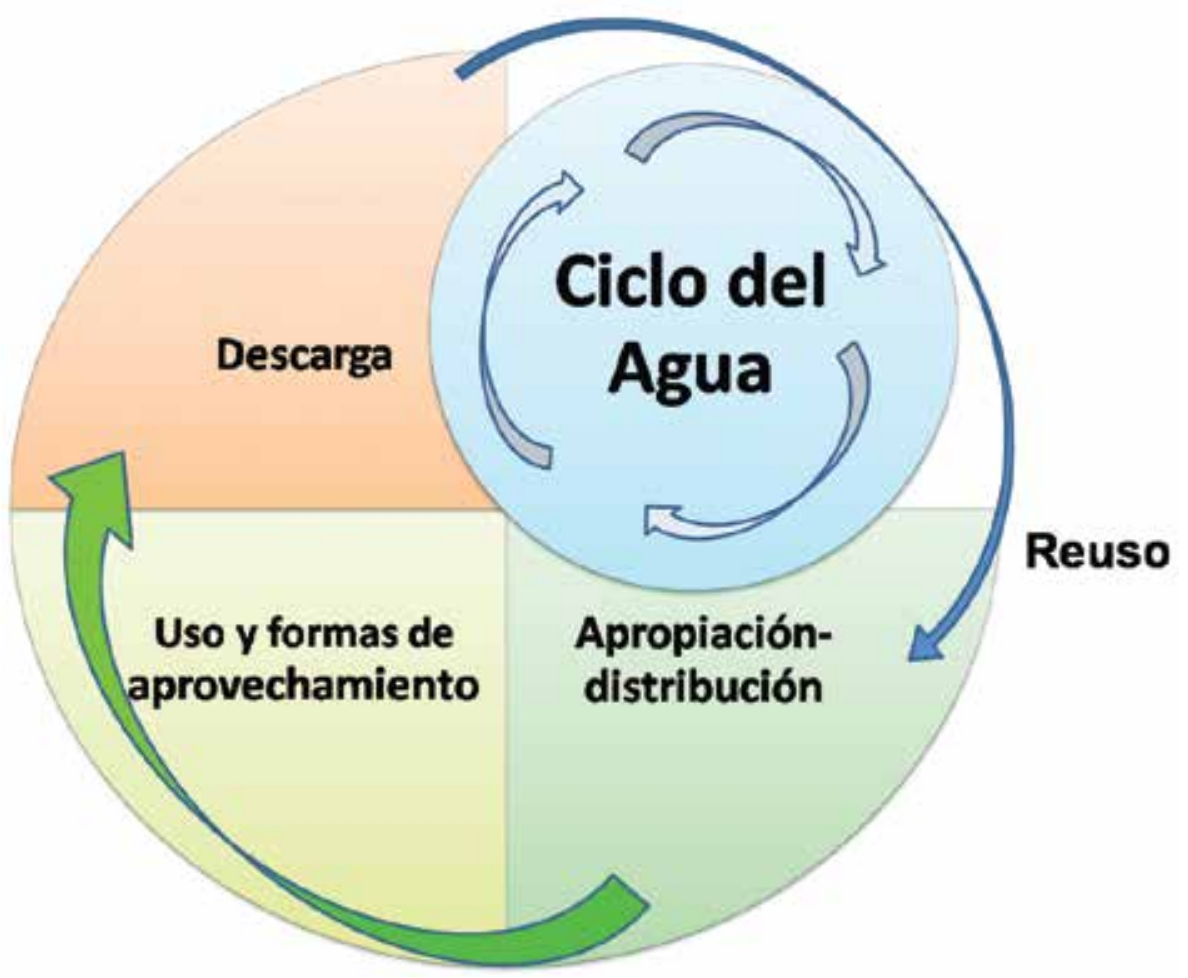

Fuente: Elaboración propia con base en Alvarado y otros (2013a)

Este proceso de manera idealizada constituye un ciclo social del recurso, cuando las aguas servidas son trasladadas a un nuevo uso, como el riego agrícola, sean o no sometidas a algún tratamiento que elimine o disminuya la cantidad de sus contaminantes; o bien, que se reincorporan al medio físico en algún cuerpo de agua.

Por tanto, las actividades de manejo de agua están destinadas al uso o aprovechamiento del recurso; sin embargo, es necesario un ente de control que administre y dirija estas actividades, y por ello el manejo queda inscrito en la gestión del recurso, para el caso de estudio, a nivel de cuenca. 
Por su parte, la "gestión" deriva etimológicamente del vocablo latín gestio, referido a la acción y efecto de administrar algo (RAE, 2014), lo que implica hacer todas las diligencias necesarias para llegar a una meta deseada. Por ello, al gestionar se realizan de todos los trámites, negociaciones y convenios entre las partes involucradas para lograr un determinado objetivo. En la figura 3 se presentan diversas formas de gestión de acuerdo al nivel de desagregación que reconocen Dourojeanni y otros (2002).

Figura 3. Jerarquización de acciones de gestión a nivel de cuencas

\section{Gestión ambiental}

\section{Gestión de recursos naturales}

\section{Gestión multisectorial del agua}

Manejo de cuencas

Gestión sectorial del agua

Fuente: Dourojeanni y otros, 2002.

En estos términos, la gestión ambiental involucra a todos los recursos naturales y la infraestructura presente en la cuenca (Dourojeanni, 2002) con todas las decisiones sociales que se ejecutan en ella. Esta forma de gestión contempla la creación, desarrollo y puesta en marcha de proyectos para el desarrollo regional con base en el aprovechamiento de los recursos naturales de la cuenca; además, contempla el mantenimiento, manejo y conservación de las obras de infraestructura.

El siguiente nivel está dado por la gestión de los recursos naturales, como el conjunto de acciones orientadas al ordenamiento, conservación y aprovechamiento de dichos recursos. Posteriormente, se encuentra la gestión del recurso hídrico y las diversas modalidades que éste tiene.

La gestión multisectorial del agua se refiere a la consideración conjunta de todas las acciones de todos los sectores, directa o indirectamente 
relacionados, en torno al recurso hídrico de manera coordinada. Implica la toma de decisiones en relación con los usos, la distribución y las metas: sociales y económicas (RIDCGIRH, 2005). La integralidad de la gestión significa la participación coordinada de todos los sectores participantes en conjunto (Dourojeanni, 2008), tomando en cuenta la influencia que cada uno tiene sobre otro y el involucramiento de los usuarios en la toma de decisiones.

En este sentido, la FAO (2007) sostiene que para realizar cualquier proceso de gestión es importante comprender la situación a gestionar como un conjunto de múltiples elementos y procesos interrelacionados. Las interrelaciones entre los subsistemas biofísicos y sociales son de gran importancia, ya que la gestión social es el camino a un desarrollo sostenible (FAO, 2007), toda vez que es la variable independiente que genera las consecuencias en los procesos sociales y naturales, que además es factible de manipular mediante la gestión.

De esta manera, el manejo y gestión del agua y de los recursos inherentes a ella, interfieren en la respuesta hidrológica de la cuenca; de ahí, la importancia de regular dichas intervenciones para obtener el desarrollo sustentable deseado en la cuenca.

De acuerdo con la Red Internacional para el Desarrollo de Capacidades en la Gestión Integrada del Recurso Hídrico (2005), la gestión integral debe alcanzar la equidad social y la eficiencia económica, haciendo partícipes a los actores y usuarios, así como a los interesados en el proceso de gestión. En este sentido, la gestión del recurso hídrico deja de ser sólo un asunto gubernamental para integrar al sector social en la toma de decisiones.

Por ello, en la conformación de un plan de gestión es importante definir el manejo que se plantea tener de cada uno de los elementos que participan en el proceso de manejo del agua, a fin de gestionar estrategias de intervención, para que las acciones que se ejecutaren cumplan con los objetivos deseados. La inclusión de la población local en el proceso de gestión facilita no sólo el diagnóstico de la problemática y de las potencialidades, sino también de la consecuente planeación de la gestión.

En ese sentido, de acuerdo a lo expuesto por Chávez y Chávez (2009), el contexto histórico del manejo hídrico, reconoce al menos tres modelos de planeación para la gestión del agua: 
a) Orientado a la oferta; supone que el volumen de agua al que se tiene acceso es suficiente y basto para cumplir con los requerimientos de la población.

b) Basado en la demanda. Tiene como único fin cubrir el requerimiento de agua de la población. No se contemplan acciones de recuperación, preservación y protección del recurso hídrico, se recurre a la explotación de nuevas fuentes de difícil y costoso acceso.

c) Integrador u holístico. Tiene como objetivo lograr la sustentabilidad en el manejo del agua y los recursos naturales, mediante el aumento de su productividad e involucra a los diferentes grupos y sectores para la toma de decisiones.

El modelo de gestión desarrollo y crecimiento económico que prevalece en México, orienta la gestión del líquido hacia la satisfacción social, y lleva cada vez mayores caudales desde sitios lejanos; deja de lado el aprovechamiento local de la precipitación, la depuración y la reutilización del agua (GEM, 2010). Ejemplo de ello es la Ciudad de México, con el trasvase de agua a través de los acueductos: Chapultepec, Xochimilco, Lerma y Cutzamala, que ha generado conflictos sociales que han resultado en la creación de grupos de defensa y protección de sus recursos naturales; mismos que se están presentando en los proyectos como Acueducto Independencia para Hermosillo, el trasvase de la cuenca del Pánuco para Monterrey, el acueducto de Chapultepec para Acapulco o las actividades de fraking y las presas que se pretenden construir en el estado de Guerrero, por mencionar algunos casos reportados por la prensa nacional y local.

De esta manera, entender, explicar y reflejar una gestión integral de los recursos hídricos y el proceso de manejo del agua, requiere identificar y categorizar la parte humana que interviene en los procesos del ciclo hidrológico, que frecuentemente son alterados, afectando al recurso como manifestaciones ambientales. Por consiguiente, conocer el manejo del agua ayuda a entender las relaciones socio-ambientales surgidas en torno a su uso y aprovechamiento.

\section{La cuenca hidrológica como unidad de gestión}

Para realizar la gestión de recursos naturales es indispensable dar una delimitación. La cuenca es una zona de la superficie terrestre, donde

Revista Geográfica de América Central No 59 ISSN 1011-484X, julio-diciembre 2017, pp. 275-307 
el agua, lluvia o nieve, se precipitan sobre ella y es drenada por un sistema de corrientes hacia un punto de salida en común (Aparicio, 2006, Díaz, Esteller y López-Vera, 2006). En este sentido, la cuenca es la unidad básica de estudio para el ciclo hidrológico y por esta razón la hace la unidad básica para la gestión de recursos hídricos (García, 2015; GFM, 2014, Balairón, 2002, Dourojeanni, Jouravlev y Chávez, 2002), puesto que se pueden compatibilizar el ciclo natural y el ciclo social del agua.

De este modo, la cuenca hidrológica es enfocada como un sistema complejo cuya función es brindar recursos para el sustento de los ecosistemas y de las sociedades humanas que se asienten en su territorio. Dentro de este último aspecto, la cuenca funge también como vertedero de los residuos líquidos que, idealmente, deberían ser saneados antes de disponernos en los cuerpos de agua.

Como sistema, la cuenca posee entradas, procesos y salidas. La precipitación, radiación solar, las actividades humanas, el crecimiento urbano, el clima, las normas, leyes y políticas para la organización, control y regulación de los procesos sociales desarrollados en la cuenca constituyen las entradas (UNAD, 2015).

Las salidas del sistema corresponden a la respuesta que se obtiene a partir de los procesos en la cuenca, como el abastecimiento de agua para uso humano, la generación de energía hidroeléctrica, la producción de alimentos, el coeficiente de escurrimiento, los arroyos, lagos y acuíferos, la contaminación, sobreexplotación y merma del agua superficial y subterránea, la pérdida de humedales, inundaciones, escasez del recurso hídrico, desertificación, pobreza y marginación social, entre otras (UNAD, 2015; Coronado, 2008).

Las salidas negativas del sistema cuenca son consecuencia de la falta de un proyecto de manejo adecuado que contemple las interrelaciones que existen entre todos los elementos y sus funciones (UNAD, 2015; Díez, 2012; Dourojeanni, 2010b). Es por ello, que el proceso de manejo del agua es un abordaje sistémico que contribuye a entender cómo la sociedad obtiene el recurso hídrico, hace uso de él y lo desecha, con una serie de consecuencias que se manifiestan como problemas ambientales y desastres no naturales. 


\section{Los Actores}

En la gestión de cuenca se considera como actor a toda persona, grupo u organización que tenga relación directa o indirecta con la administración, manejo o uso del recurso hídrico (CONAGUA, 2012). La relación que tenga el actor con el recurso y con el resto de los actores, define la forma de aprovechamiento o uso del recurso, la toma de decisiones en torno a él y el futuro del mismo. De acuerdo con esto, los actores pueden tener poder político, económico o social, de manera que puedan estar a favor o en contra de propuestas, y así intervenir en cualquier sociedad o territorio.

De esta manera, para el caso de estudio se distinguen dos tipos de actores: los sociales y los políticos. Es importante diferenciar al actor social del actor político, puesto que sus atribuciones y funciones son desarrolladas en un ambiente normativo específico definido por el conjunto de normas y leyes concretas. Las limitantes que enfrentan cada uno de los actores, establecen la forma en que estos afrontan una problemática en particular, tales como lo son el poder político y social, los recursos con que cuentan, e incluso el territorio de su injerencia.

\section{Actores sociales}

Los actores sociales son los grupos organizados que en gran medida están conformados por los usuarios (Oré y Geng, 2010) para el caso del agua. Emergen en el contexto de derecho a la participación social, cuyo objetivo es modificar su realidad sin la necesidad de pertenecer a un aparato gubernamental; en algunos casos buscan mediante su iniciativa el mejoramiento de las condiciones socio-económicas de la población (Dávila, 1996,1993).

Las organizaciones comunales son actores sociales con mayor peso en el ámbito municipal; también existen las organizaciones de vecinos o de colonia, los líderes de manzana, los delegados municipales, etc., quienes externan las necesidades de algún sector de la población a la autoridad municipal para buscar una solución. Este sector comprende a las organizaciones no gubernamentales, cuyos propósitos y actuación dependerán de los intereses de la fundación. 


\section{Actores políticos}

Los actores políticos se encuentran representados en los organismos gubernamentales, las instituciones públicas y los partidos políticos (Oré y Geng, 2010). Un actor político es toda persona u organización pública con la capacidad de administrar, manejar e impulsar algún plan, programa o proyecto por parte de cualquier orden de gobierno.

Se consideran como actores políticos a las autoridades y dirigentes políticos que forman parte de un ente de gobierno, así como el personal especializado y técnico que labora con ellos. Están relacionados directamente con las decisiones político-administrativas de su jurisdicción; su poder es variable, algunos poseen facultades legales y sociales para ejercer sus atribuciones como administradores de los bienes públicos, por lo que sus decisiones marcan el rumbo en el desarrollo social, económico y político.

En un sistema democrático, los líderes sociales se pueden convertir en actores políticos mediante su elección para ocupar un cargo gubernamental; de este modo, las necesidades y problemas de la comunidad pueden ser fácilmente entendidos y atendidos. Sin embargo, la corrupción y los intereses propios de los políticos empañan esta situación, lo que ocasiona rezago social.

Los actores políticos y sociales se encuentran en una dinámica en la que ambos conocen sus funciones y el entorno en el que coexisten. Por esta razón, ambos deben estar involucrados en la toma de decisiones para la gestión de los recursos naturales, con el fin de realizar acuerdos que favorezcan el desarrollo sustentable del territorio (Oré y Geng, 2010).

\section{Marco metodológico}

Para plantear una propuesta metodológica para la gestión de la MM se procedió en dos modalidades: el trabajo de gabinete y el trabajo de campo. La investigación inicia con la delimitación y descripción de la zona de estudio a partir de cada una de las cinco dimensiones o subsistemas, con la finalidad de comprender el proceso de manejo del agua en la microcuenca. Las cinco dimensiones del proceso de manejo del agua son: Biofísica, Económica, Sociocultural, Tecnológica y Político-Administrativa.

Se recurrió a los métodos de análisis y síntesis, el primero de los cuales tiene la finalidad de segmentar y estudiar individualmente cada subsistema y sus componentes, a fin de conocer los elementos de cada uno de 
ellos y caracterizarlos, de manera que con el método de síntesis se pueda entender en conjunto el sistema cuenca y el sistema de gestión.

Posteriormente, a partir de una serie de programas gubernamentales para el saneamiento del agua, se profundizó en la dimensión político administrativa, con el fin de centrarse en la metodología de gestión que se propone para el proyecto "Tubos y plantas de tratamiento". En este sentido, se realizó la búsqueda y recopilación de información bibliográfica sobre normatividad y legalidad en materia de saneamiento de agua, y trabajo de campo, basado en las metodologías propuestas por Cano (2004), Pozo (2007), Tapella (2007), para la identificación y análisis de actores.

A partir del conocimiento de la zona de estudio y en base a la propuesta de solución se consultaron leyes y programas de los tres órdenes de gobierno, en los que se pudiera inscribir el proyecto de saneamiento; además, de acuerdo a las metodologías estudiadas, se conformó una propuesta de gestión de recursos hídricos a nivel de cuenca para el caso de la MM.

Posteriormente, de acuerdo a base a la legislación mexicana, se identificaron a los actores políticos que participan en el saneamiento del agua. En cambio, los actores sociales se identificaron a través del trabajo de campo por medio de la entrevista a autoridades municipales y locales. Con los actores identificados, se realizaron entrevistas no estructuradas para conocer la percepción de cada actor frente al proyecto de "Tubos y plantas de tratamiento" (a favor, en contra o indiferente), y algunas características de su organización (intereses, cooperación, recursos, dependencia, objetivos, influencia). Con la información registrada se identificaron las posibles afectaciones, resistencias, metas e intereses de los actores, y a partir de estos diseñar una estrategia que se acerque a la implementación del proyecto "Tubos y plantas de tratamiento".

Con los resultados se elaboró el mapa de actores mediante el método MACTOR (Matriz de Alianzas y Conflictos: Tácticas, Objetivos y Recomendaciones) propuesto por Cano (2004) y que consiste en la representación gráfica de la dinámica de actores, el cual contribuyó al planteamiento de la propuesta de gestión en el proyecto "Tubos y Plantas de Tratamiento" para el saneamiento de la MM. 


\section{Resultados}

El marco en el que suceden los procesos de contaminación y de gestión del agua queda definido por la cuenca hidrológica, la cual se describe mediante los subsistemas que la conforman; además, esta es parte integrante del proceso de manejo del agua y es el espacio donde se desenvuelven los actores, centrados de manera particular en el subsistema político-administrativo, en conjunto con los lineamientos de gestión y la propuesta metodológica para la gestión integral de microcuencas.

\section{Los subsistemas de la Microcuenca del Muerto Subsistema Biofísico}

La MM se encuentra en la vertiente Occidental de la sierra de Las Cruces, tiene como corriente principal a El Muerto, que es una corriente de tercer orden. Dicho arroyo tiene su inicio en el cerro El Muñeco a 3,750 msnm y desemboca en la laguna de Almaya a los 2720 msnm, con presencia de montañas, lomeríos y pequeños valles intermontanos.

Son tres los asentamientos humanos que realizan su proceso de manejo del agua dentro de la microcuenca, al menos parcialmente, los cuales son, en orden descendente altitudinal: San Pedro Atlapulco, perteneciente al municipio de Ocoyoacac; el paraje conocido como Valle de El Potrero, el cual forma parte de la zona turística La Marquesa; y Guadalupe Victoria, que se ubica en el municipio de Capulhuac.

La MM es parte dela provincia fisiográfica del Eje Neovolcánico, por lo que destacan los materiales de esta formación geológica y su trascendencia en la geohidrología regional. La precipitación anual en la zona se encuentra entre 858 y $1307 \mathrm{~mm}$, según la altitud, presentando una distribución temporal que la concentra en un promedio del $82 \%$ durante el semestre de mayo a octubre (Alvarado y otros 2013b).

Dentro de los usos del suelo, destaca principalmente el boscoso (46.24\%) seguido por el agrícola (35.10\%), con amplia presencia de bosque en más de la tercera parte, sobre todo en las cimas de las montañas. En estas condiciones, la respuesta hidrológica superficial de la microcuenca es un caudal permanente, que fluctúa entre $\operatorname{los} 100$ a $150 \mathrm{~m}^{3} / \mathrm{s}$ (Alvarado y otros 2013b) y es alimentado por manantiales y aguas residuales domésticas, generadas por las viviendas y el Valle de El Potrero. Dicho caudal cruza una zona con un coeficiente de escurrimiento del 15\% (INEGI, 2016). 
Esta característica contribuye a la recarga del acuífero del Valle de Toluca, que abastece al valle del mismo nombre, y parcialmente al Valle de México (Alvarado y otros 2013b). Es por ello, que el manejo del territorio en la zona trasciende el interés local.

\section{Subsistema Económico}

Entre las actividades económicas que se realizan en la MM destaca la venta de los servicios turísticos en los valles intermontanos, pero también existe la agricultura de temporada, la cosecha de champiñones, la producción de trucha, la ganadería, la forestería comunitaria como herramienta en el manejo sustentable del bosque, y la producción de artefactos pirotécnicos.

Las actividades agrícolas que se realizan son en su mayoría para el autoconsumo, el resto son comercializados en la zona turística o en los poblados cercanos. Los principales cultivos son de maíz, avena, haba, zanahoria y papa, para los cuales los pobladores recurren principalmente al uso de agroquímicos y fertilizantes naturales como el estiércol.

El comercio de mercancías manufacturadas fuera de la zona es una actividad relevante en los pueblos que antes fueron arrieros; sin embargo, la principal actividad que se relaciona con el manejo del agua es el uso doméstico con el empleo combinado de diversos productos para el aseo y la cocción de alimentos, los cuales contienen sustancias químicas que contaminan al agua por las actividades humanas.

\section{Subsistema Tecnológico}

En el proceso de manejo del agua es importante considerar la accesibilidad de la población al adquirir el recurso y la forma en que éstos disponen sus desechos. En este sentido, la tecnología con que cuenta una comunidad define los hábitos de los usuarios, los cuales reinciden en la cantidad, calidad y disponibilidad del agua y su saneamiento.

La MM es abastecida con agua proveniente de los manantiales perenes a través de la instalación de tubos de polietileno de alta densidad, de diámetro variable. Después de su uso en las viviendas, centros de venta de alimentos y estanques acuícolas, principalmente, el agua es descargada al cauce del arroyo y a la red hidrológica de la microcuenca sin tratamiento; las aguas que no se infiltran en el trayecto son conducidas hacia la laguna de Almaya.

Revista Geográfica de América Central No 59 ISSN 1011-484X, julio-diciembre 2017, pp. 275-307 
Otra actividad que hace uso del agua, pero sin infraestructura, es la agricultura, la cual genera traslado de agroquímicos por las prácticas productivas; estas prácticas contribuyen a la eutrofización del cuerpo de agua.

Por su parte, la red de distribución de agua en San Pedro Atlapulco presenta fugas en varios puntos, reduce la eficiencia del sistema y provoca pérdidas de volumen disponible en su trayecto. Además, ninguna autoridad da seguimiento al estado de las redes de agua y alcantarillado, no tienen registro de los caudales de cada red para detectar fugas y, en general, desconocen el estado del sistema por lo que el mantenimiento sólo es correctivo.

Al problema de contaminación del agua, por cualquiera de sus usos, se suma su contaminación por manejo de residuos sólidos, ya que se encontraron tiraderos clandestinos, al aire libre, que provocan su traslado y el de lixiviados.

\section{Subsistema Sociocultural}

Se tiene el registro que desde el año 650 d.C. existieron asentamientos humanos en la parte alta de la MM, formados por grupos indígenas de otomíes y matlatzincas (Ocoyoacac, 2013). Uno de esos asentamientos desapareció por la fuerza del agua; los sobrevivientes fundaron nuevamente el poblado en el lugar donde se encuentra San Pedro Atlapulco, conservando hasta el día de hoy rasgos de su cultura prehispánica (mayo, 2007), que se manifiesta por los usos y costumbres de la población de la zona. En este contexto, los pobladores preservan el bosque de oyamel y son favorecidos con los bienes ambientales que produce.

En contraste, la comunidad de Guadalupe Victoria es un poblado sin raíces prehispánicas, pero tienen un fuerte sentido de identidad con el pueblo. La autoridad local está representada por los delegados municipales.

Con base en el censo de población y vivienda del año 2010, Guadalupe Victoria, se considera un asentamiento rural, ya que no rebasa los 2,500 habitantes; San Pedro Atlapulco contó con 4,288 habitantes (INEGI, 2010). Los servicios básicos de agua potable, drenaje y electricidad cubren casi la totalidad de las viviendas en la microcuenca y la ocupación es de 4 habitantes en promedio por cada hogar (INEGI, 2010).

El nivel de estudios de la población en ambas localidades es de un promedio de 9 años para las personas con al menos 18 años cumplidos, lo que equivale a la educación básica en México. Esto da una idea de las 
capacidades derivadas del nivel de escolaridad de la población para enfrentar diversas problemáticas. Además, existe un nivel de analfabetismo de $4.32 \%$, similar al del estado de México que es de $4.38 \%$ (INEGI, 2010).

Además del bajo nivel de escolaridad, que en general existe en la microcuenca, y de los antecedentes prehispánicos con los que cuenta, la educación ambiental es precaria, puesto que la información con que cuentan los pobladores hace difícil que los niños y jóvenes aprendan y adopten las herramientas necesarias para el manejo de los recursos naturales.

\section{Subsistema Político-Administrativo}

Este subsistema es el componente principal de la investigación, ya que en él se encuentran los actores para la gestión de la microcuenca y el marco normativo que rige los alcances de los lineamientos de gestión. Por tanto, es en este subsistema donde se gestan las decisiones y acciones en materia de saneamiento del arroyo El Muerto y su cuenca.

En la MM se encuentran dos localidades, cuyos orígenes y forma de administración son diferentes, donde conviven los usos y costumbres prehispánicos de los pueblos indígenas con la estructura administrativa municipal y las autoridades cívico-religiosas. Sin embargo, cada una tiene identidad y autoridad que les permite ser parte de la gestión de sus recursos.

La presencia de diferentes autoridades provoca una composición, en la que coexisten las estructuras sociales y políticas que funcionan gracias a la aceptación y reconocimiento de la población civil. En este sentido, es posible establecer una propuesta de gestión participativa para dar solución al problema de contaminación del agua en la microcuenca de El Muerto.

\section{Los actores de la microcuenca del arroyo EI Muerto}

Para el caso de la MM se identificaron diversos actores, los cuales se clasificaron de acuerdo a la escala geográfica en la que interceden y la tipología social o política en la que se encuentran inscritos:

\section{a. Actores Políticos}

A partir de la revisión del marco jurídico, se identificaron los actores políticos que establece la normatividad. A partir de las asignaciones que 
cada orden de gobierno tiene en relación con el saneamiento del agua, se identificaron a los siguientes:

\section{a) A nivel federal}

Le compete al Ejecutivo Federal la concesión para el uso y aprovechamiento de los recursos naturales. Sus atribuciones las realizará mediante la Secretaría de Marina y Recursos Naturales (SEMARNAT) y la Comisión Nacional del Agua (CONAGUA), acatando lo que establece el Gobierno Federal Mexicano (2014a, 2014c) como cabeza del sector hidráulico nacional. Entre sus atribuciones destaca regular la explotación, uso, aprovechamiento, distribución, control, preservación en calidad y cantidad de las aguas nacionales, para lograr su desarrollo integral sustentable, toda vez que el agua es propiedad de la Nación (GFM, 2014a: art. 27).

\section{b) A nivel Regional}

Con el fin de cumplir con sus atribuciones, la CONAGUA creó trece Organismos de Cuenca como unidades autónomas, técnicas, administrativas y jurídicas especializadas, que vinculan directamente al titular de CONAGUA con los Consejos de Cuenca para desarrollar sus funciones de manera conjunta (García y Galindo, 2015; GFM, 2014b). Los Consejos de Cuenca son órganos consultivos coordinados donde intervienen representantes gubernamentales y de la sociedad, es decir, son de integración mixta. Cuentan con diversos comités, según las características de la cuenca hidrológica en cuestión, los cuales no se encuentra subordinados a la CONAGUA o al Organismo de Cuenca correspondiente.

Entre el Consejo y el Organismo de la Cuenca del Balsas desarrollan acuerdos sobre manejo y gestión en la región donde se ubica la MM. Además, el Organismo de cuenca está relacionado con la Comisión para la Recuperación de la Cuenca del Río Lerma. Éste último organismo tiene a su cargo a la Comisión para la Recuperación Ecológica de la Cuenca Alta del Río Lerma, fracción correspondiente a la zona de la MM, quien colabora con el gobierno estatal.

Por otra parte, el Gobierno del Distrito Federal interviene a través del pago de servicios ecosistémicos por el agua que la microcuenca suministra a la capital del país. Entre los trabajos que ha realizado con los pobladores se encuentra la construcción del sistema de drenaje, separado 
en parte de la población de Atalpulco; sin embargo, la falta de instrucción sobre su funcionamiento ocasionó que la obra fuera ineficaz.

\section{c) A nivel estatal}

La Secretaría del Agua y Obra Pública SAOP del Gobierno del Estado de México, a través de la Comisión de Agua del Estado de México CAEM y de la Comisión Coordinadora para la Restauración Ecológica de la Cuenca del Río Lerma CCRCRL, coordinan la administración de los recursos hídricos del Estado de México. De manera análoga a la CONAGUA, la Comisión del Agua del Estado de México CAEM es el organismo público descentralizado de la Secretaría del Agua y Obra Pública del Estado, quien cuenta con personalidad jurídica y patrimonio propios en materia de agua (CAEM, 2001).

Le corresponde a la CAEM planear, programar, presupuestar, diseñar, construir, conservar, mantener, operar y administrar los sistemas de suministro de agua potable, desinfección, drenaje, alcantarillado, saneamiento, tratamiento y reúso de aguas tratadas, así como la disposición final de sus productos resultantes; impone las sanciones que correspondan en caso de incumplimiento de la normatividad en materia de agua (GEM, 2011).

Actualmente la CAEM tiene entre sus proyectos la "Planeación General de Actualización del Proyecto Ejecutivo del Sistema de Alcantarillado Intermunicipal Jalatlaco, Capulhuac y Tianguistenco, Estado de México", inédito y sin fecha, en el que se marcan los colectores de los municipios mencionados, sin ningún beneficio a la laguna de Almaya y a los pobladores aguas arriba de la misma.

\section{d) A nivel municipal}

San Pedro Atlapulco y Guadalupe Victoria están regidas bajo el esquema municipal político representado por el Delegado de la localidad como autoridades auxiliares del municipio correspondiente. Entre sus funciones se encuentra vigilar el estado de los canales, colectores, barrancas, canales de alcantarillado y desagües, además de informar al ayuntamiento sobre las acciones necesarias para la funcionalidad de los mismos (GEM, 2015). Los trabajos realizados por los delegados no son retribuidos por la ley, por lo que su tiempo está destinado a obtener recursos para su propia manutención; el tiempo libre del que disponen lo destinan para sus funciones.

Revista Geográfica de América Central No 59 ISSN 1011-484X, julio-diciembre 2017, pp. 275-307 
Además del delegado, San Pedro Atlapulco, cuenta con el Consejo de Participación Ciudadana (COPACI), otra autoridad auxiliar del municipio reconocida por la ley estatal (GEM, 2015). Este Consejo está conformado por tres titulares (un presidente, un secretario y un tesorero) y dos suplentes, los cuales son electos por la población mediante sufragio.

El COPACI colabora con el delegado para atender las necesidades de la población referente a los servicios públicos municipales que incluyen: agua potable, alcantarillado y saneamiento hídrico. Sus funciones las desempeña con apoyo de la organización de los ciudadanos para involucrarlos mediante faenas, las cuales se realizan por convocatoria abierta y su fin es mantener en el mejor estado posible las instalaciones de los servicios públicos de la comunidad. Este tipo de trabajo comunitario ha dado buenos resultados, puesto que la población responde activamente; sin embargo, la falta de recursos económicos limita el alcance de los trabajos tanto en cantidad como en calidad.

\section{b. Actores sociales}

a) En el de poblado de San Pedro Atlapulco

El poblado de Atlapulco adquirió la restitución de sus tierras y agua gracias a la Reforma Agraria en 1946, por lo que los dominios están bajo el esquema de tierras comunales. Esta característica produce grandes beneficios a la comunidad, como el control para el aprovechamiento de sus suelos y manantiales, puesto que las decisiones se toman de manera conjunta entre los pobladores en la Asamblea General del Pueblo.

En contraste, Guadalupe Victoria no se encuentra en tierras ejidales o comunales. En tal sentido, la tenencia de la tierra en la zona es particular y significativa al definir la estructura de gobierno y del poder político y social.

\section{b) En la comunidad agraria}

El Comisariado de Bienes Comunales de San Pedro Atlapulco es la autoridad agraria que tiene control sobre las tierras y está formado por un presidente, un secretario, un tesorero, sus respectivos suplentes, y propietarios. Dichos cargos son elegidos por la comunidad y entre sus funciones está la gestión de las tierras y los recursos que en ella se encuentran.

El territorio comunal fue distribuido básicamente en dos secciones: el área agrícola y el área boscosa (4600 ha aprox.). La estructura de las 
tierras agrarias la forman: tierras parceladas de uso común y el casco urbano. El bosque se encuentra bajo el régimen de manejo forestal sustentable y se realizan actividades de protección, restauración, conservación, cultivo y aprovechamiento de recursos forestales maderables.

Los acuerdos y decisiones son tomados en reunión plenaria, de la cual surgen las actividades designadas al Comisariado de Bienes Comunales en lo que respecta al manejo de los bienes comunales. Para realizar dichas actividades y cumplir sus funciones, el Comisariado obtiene ingresos económicos del pago que realizan los visitantes a los centros recreativos de los valles, del cobro por explotación minera y del pago de los servicios ambientales generados, como la explotación forestal y del agua de los manantiales.

c) A nivel del Valle del Potrero

El Valle del Potrero es una zona de turismo campestre, la cual es administrada por el consejo del mismo nombre. Conformado por comuneros, es una organización formada por un presidente, un secretario, un tesorero, de cuatro a seis vocales y sus suplentes, quienes tienen algún tipo de comercio dentro del valle.

El Consejo tiene entre sus responsabilidades cuidar y mantener el sistema ecológico de todo el valle, prestar los servicios turísticos, así como el cobro de los mismos. Con quien colabora y a quien entrega cuentas de sus labores, es al Comisariado de Bienes Comunales, el cual se manifiesta sobre las actividades prioritarias a realizar en el valle en consenso con el Consejo.

Los recursos económicos con los que cuenta el valle son destinados prioritariamente al mantenimiento e imagen de la zona, ya que representa el ingreso de muchas familias. En este sentido, el proyecto que se planea implementar, el cual consiste en un sistema de tratamiento de aguas residuales de uno o varios humedales artificiales, contribuirá al mejoramiento de los arroyos y a la economía de las personas, pues está diseñado como un proyecto sustentable, del cual se pueden obtener beneficios económicos.

\section{c. Análisis de los actores}

A través de las entrevistas no estructuradas que se realizaron a los actores, se pudieron conocer sus intereses en el proyecto de saneamiento, los recursos con los que cuentan, los problemas que perciben para actuar

Revista Geográfica de América Central No 59 ISSN 1011-484X, julio-diciembre 2017, pp. 275-307 
o no en el proyecto, el nivel de prioridad que catalogan para dar solución al problema de contaminación de los arroyos, entre otros. En la figura 3 se presentan el mapa de actores.

Figura 3. Mapa de actores para el saneamiento de la microcuenca del arroyo El Muerto

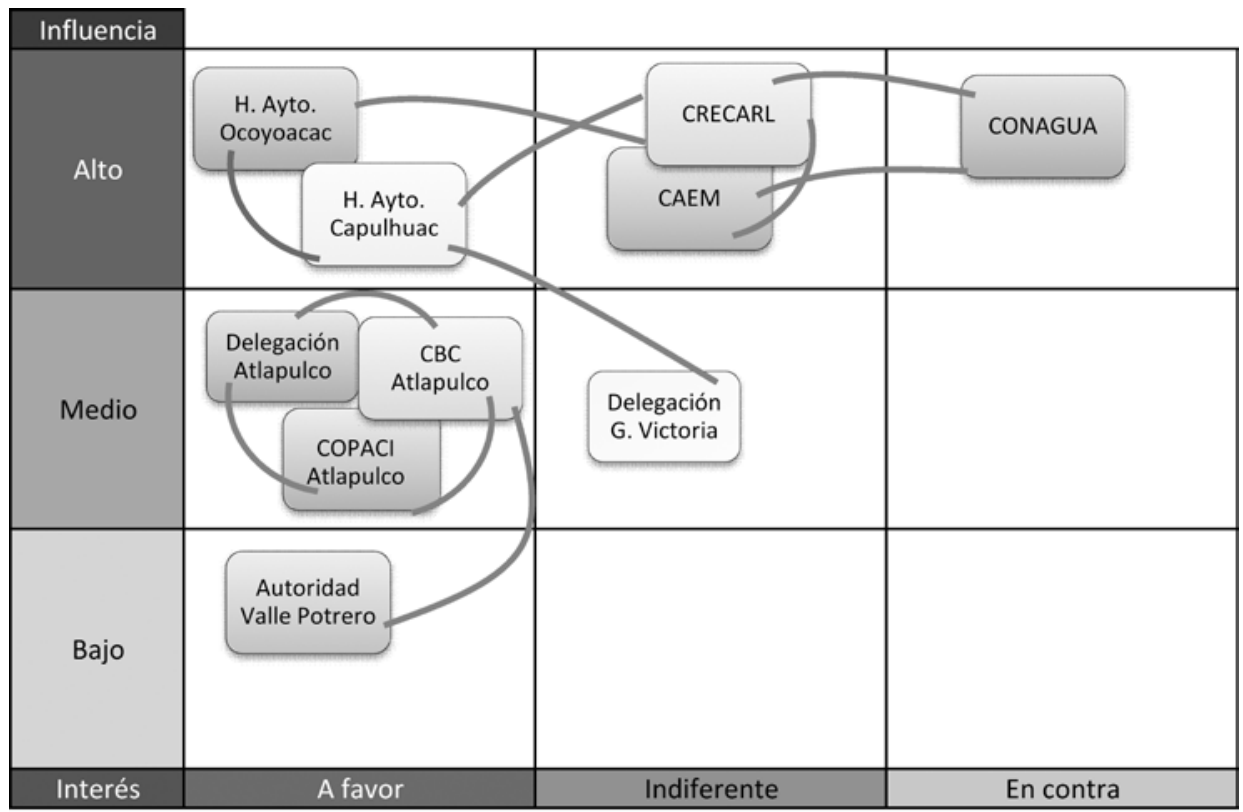

- Relación de cooperación

Relación de conflicto

La influencia alta, media o baja, representa las capacidades con que cuenta el actor para llevar a cabo el proyecto de saneamiento propuesto. Se consideraron sus atribuciones legales, sus recursos económicos y la capacidad de afectar a otros actores.

A pesar de que las autoridades auxiliares municipales y los actores sociales locales expresan gran interés en el proyecto de saneamiento, cuentan con pocos recursos económicos para dar marcha a los trabajos; sin embargo, poseen otros insumos como la propiedad de la tierra y mano de obra para participar en el proyecto. 
Existen relaciones de conflicto entre comunidades de los municipios de Ocoyoacac y Santiago Tianguistenco (donde se encuentra la laguna de Almaya) por el arrastre de contaminantes de aguas arriba de la cuenca, además, existen reclamos de los habitantes para regular el sistema de drenaje por temor a desbordes e inundaciones. Por ello, la gestión a nivel de la MM a través del programa de saneamiento pretende disminuir este tipo de conflictos, a partir de sus planteamientos basados en un enfoque holístico.

A pesar de ser los responsables, en relación a proporcionar un ambiente sano para el ser humano, los planes y proyectos de gobierno estatal y federal destinan sus esfuerzos a proyectos de gran extensión, por lo que las microcuencas y los poblados rurales muchas veces quedan fuera de ellos. El poder económico y político con el que cuentan es enorme, lo que los coloca como actores con gran influencia en el proyecto.

Por ello, es importante incumbir a estos actores políticos en proyectos de iniciativa social, y así contar con los recursos necesarios para su cumplimiento, lo que además podría generar más iniciativas por parte de la sociedad civil. Es por esta razón que el poder de influencia de los actores locales se encuentra en el nivel medio, ya que pueden intervenir con la población, pero la falta de dinero puede hacer que el proyecto no se realice.

Para enfrentar la falta de recursos financieros es importante incluir a las autoridades municipales, estatales y federales, ya sea en especies con materiales para construcción, con personal especializado para asesoramiento o con recursos económicos.

En el mapa de actores se puede observar que las autoridades estatales y federales se encuentran en la parte baja de interés ya que, aunque expresan interés en el saneamiento, no contemplan entre sus trabajos proyectos como el que se intenta implementar en la microcuenca; de igual manera, expresan que es importante incluir en la planeación y gestión de los recursos naturales a los actores sociales, pero esto sólo se queda en el discurso, pues la forma en que atienden las necesidades de las comunidades es enfocado a la oferta.

En el caso particular de la CAEM, se han llevado conversaciones entre ésta y el grupo de delegados de las localidades y sus autoridades agrarias para exponer su interés en el mejorar las condiciones ambientales de la zona. 
El delegado de Atlapulco expresa que no percibe interés en los representantes de la CAEM y que sólo postergan la revisión y la observancia de las propuestas realizadas, lo que ocasiona que las intenciones de las autoridades locales y las organizaciones sociales se diluyan.

A pesar del panorama complicado que las autoridades de los gobiernos federal y estatal presentan para el desarrollo de proyectos sociales, el trabajo con las autoridades locales es el camino que se deberá seguir para la implementación de la propuesta de la UAEM y con ello demostrar que la gestión social del agua es una opción a la privatización de los recursos hídricos.

Otra fuente de recursos económicos se encuentra en el sector privado; la Cámara Nacional de la Industria de Transformación (CANACINTRA) ha expresado que está interesada en participar en proyectos sociales, por lo que hay una oportunidad de encontrar financiamiento con esta organización.

\section{Propuesta de metodología de gestión para la implementación de un proyecto técnico de saneamiento.}

La metodología que se propone está basada en 5 etapas. Las decisiones que se tomen y acciones que se realicen en cada una de ellas afectarán a todo el modelo de gestión.

\section{El proceso de la metodología}

En la figura 4 se presenta de manera esquemática la propuesta metodológica de gestión, donde resalta el conocimiento del proceso de manejo del agua en la microcuenca como un aspecto esencial. En cada una de las etapas se debe dar continuidad a la generación de información, la participación de los interesados, y no se pueden perder de vista todos los entornos en los que se puede trabajar. 
Figura 4. Propuesta metodológica para la gestión del agua en la MM

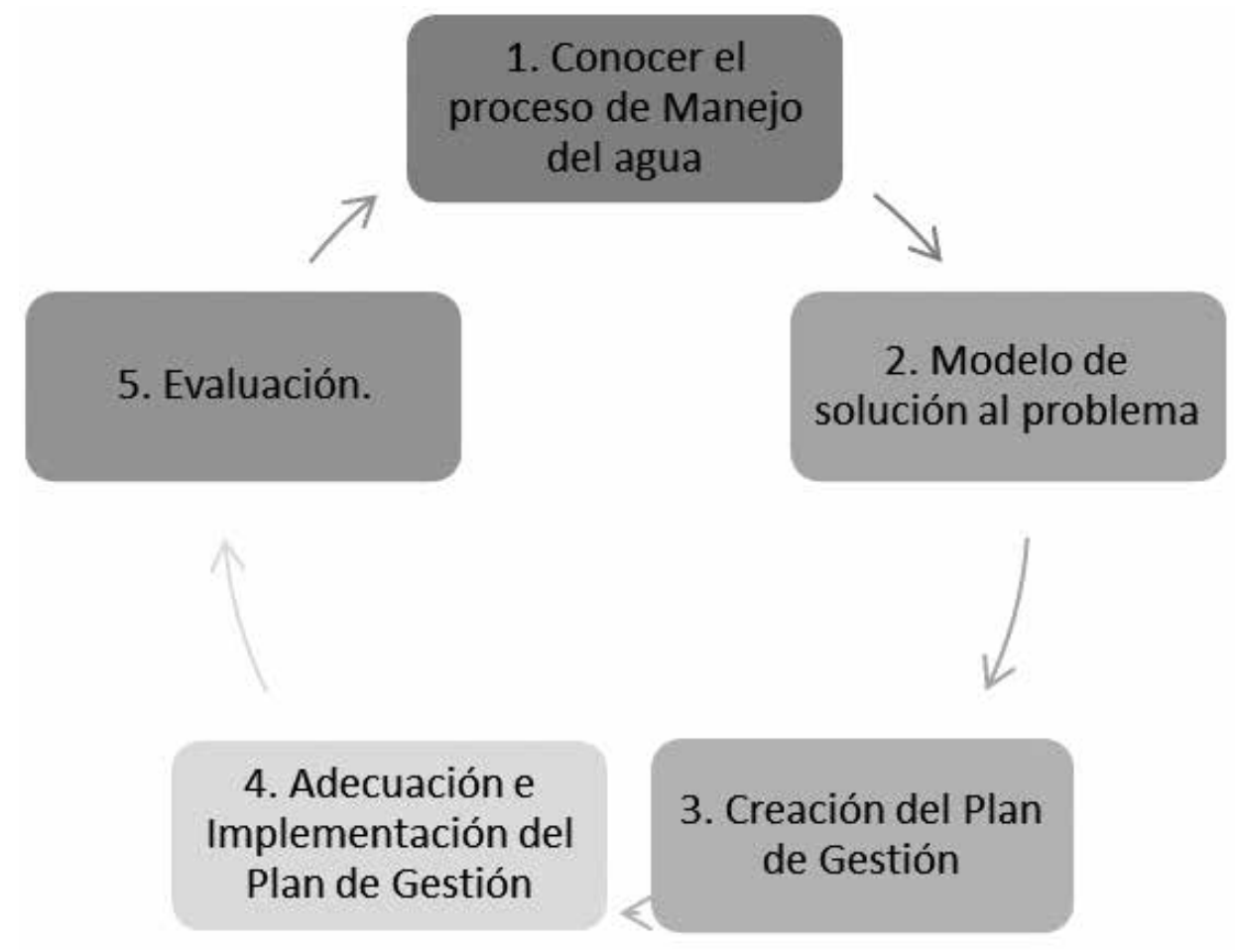

Fuente: Elaboración propia

1. Conocer el proceso de manejo. El conocimiento y comprensión del proceso de manejo del agua llevado a cabo en la cuenca servirá para distinguir el origen del problema, cuya solución es el propósito del plan de gestión. En este paso se concentran los estudios y el diagnóstico de la cuenca respecto al proceso de manejo del agua.

2. Modelo de solución al problema. Después de conocer y comprender el proceso de manejo del agua en la cuenca, es posible identificar cuál es el origen del problema, y de ese modo aportar una solución.

3. Creación del plan de gestión. Deberá contemplar la participación de todos los interesados y los que de alguna manera puedan aportar a la solución del problema. Antes se debe determinar quiénes serán y cómo será su participación. Por ello, es importante identificar a los actores y los programas existentes que puedan apoyar la implementación del proyecto. Conocer el marco legal y normativo proporcionará en una primera instancia quienes son los 
actores involucrados en el manejo del recurso hídrico, y en segunda instancia dará cuenta de las competencias y atribuciones de cada actor. El acercamiento a ellos mediante entrevistas dará una idea de qué tan (involucrados o comprometidos) están con sus facultades, derechos, obligaciones y actividades; incluso, podrán dar a conocer otros actores con los que se relacionan.

Es importante definir quienes conformarán el grupo de control y vigilancia del proceso del plan de gestión, además de asignar a cada actor su rol de participación.

4. Adecuación e Implementación del plan de gestión. Esta etapa involucra la acción de todos los actores, cuyas labores darán rumbo al proyecto. En este paso se podrá evaluar y adecuar el plan de gestión planteado, de acuerdo a los comentarios de los participantes en las reuniones que se convoquen. El objetivo es la aprobación y admisión del proyecto por parte de todos los actores.

5. Evaluación. Aunque parezca el último paso, la evaluación y vigilancia del proceso de gestión se debe hacer de manera continua desde su implementación, puesto que es importante no perder el control sobre el plan de gestión y hacer las adecuaciones necesarias para llegar al objetivo planteado.

Los pasos del uno al tres han sido realizados por el grupo de investigadores mencionado en este artículo. La siguiente etapa consiste en llevar a ejecución los dos pasos restantes, con el fin de contribuir con el saneamiento de la MM.

\section{Lineamientos de gestión para la MM}

De la investigación realizada, surgieron los lineamientos a seguir con el proyecto de saneamiento de la microcuenca. En primer término, ya que los comuneros, el delegado de San Pedro Atlapulco y el de Guadalupe Victoria han expresado su interés en la implementación del proyecto, es importante que el resto de la población lo acepte también, y así continuar con la generación del proyecto ejecutivo de las obras necesarias. En este sentido, es importante mantener comunicación con los actores sociales para dar continuidad al proyecto.

El convocar a reunión a todos los actores participantes en el saneamiento del agua de la microcuenca es de vital importancia, pues de esta manera se establece de manera cercana y directa la comunicación entre ellos, y se comienza a plantear una estrategia de gestión. 
Así mismo, es importante comenzar con el proyecto ejecutivo de las obras necesarias para el saneamiento, por lo que se deberá iniciar con definir el lugar donde se instalará la planta, o las plantas, de tratamiento. Para tal fin, se deberá considerar el régimen de suelo y hacer las diligencias necesarias para el desplante del proyecto.

De las reuniones que se realicen entre los actores, se deberá establecer a la persona o grupo responsable de la gestión de los trabajos, del mismo modo, plantear los trabajos que cada uno tendrá a su cargo y la forma en que éstos se realizarán.

En este sentido, la Universidad Autónoma del Estado de México, por medio del programa mencionado al principio de este artículo y a través del cuerpo académico "Desarrollo, ambiente y procesos de configuración territorial", trabajará para dar apoyo técnico y educativo, principalmente, para trabajar con la población sobre educación ambiental y técnicas sustentables de manejo de residuos sólidos y líquidos.

Además de estas actividades, es importante recurrir a otros actores de la iniciativa privada para fortalecer el proyecto.

\section{Conclusiones}

El proceso de gestión de recursos hídricos ha cambiado durante el paso de varias décadas. Entre sus etapas, comenzó con la necesidad de abastecer de agua a la población, luego pasó por los planes formulados por ingenieros forestales, hasta llegar a involucrar a numerosos grupos sociales y políticos en la búsqueda de una gestión integral.

La gestión de recursos hídricos se ha convertido en un proceso complejo tanto desde la perspectiva ecosistémico como desde la particularidad que cada cuenca representa. Estudiar y entender los procesos que se entrelazan entre las diferentes esferas (socio-ambientales, político-sociales, etc.), forma parte del pedestal de la gestión integral de cuencas hidrológicas y de recursos hídricos.

De manera particular, el caso de estudio de MM implicó no sólo el conocimiento de los procesos hídricos de la microcuenca, sino también de las diferentes formas de uso y administración que constituyen el componente sociocultural; de ésta última, destaca que existe otra forma de realizar gestión además de la gubernamental: la gestión social. 
De esta manera, la participación activa de la población local es un brazo que sirve de soporte para la generación y puesta en marcha de proyectos sustentables; sin embargo, en muchos casos se tiene en el olvido y hasta relegada por parte del sector gubernamental, que no cuenta con proyectos para el saneamiento de la microcuenca. A pesar de esto, las autoridades agrarias que padecen el problema, demuestran interés en darle solución con sus propios medios, si es necesario, puesto que el turismo en la zona de estudio es el sustento de la población en general y es estímulo para el saneamiento hídrico por parte de los actores locales.

Con la aceptación del proyecto de saneamiento por parte de los actores sociales locales se da inicio a una nueva etapa en el desarrollo del proyecto planteado por el cuerpo académico de la Facultad de Planeación Urbana y Regional de la UAEM, el cual busca la implementación del proyecto, y ser ejemplo para otras comunidades.

Ante esta situación, es importante contemplar que las personas responsables de los servicios urbanos tengan documentadas las condiciones de diseño e instalación de las infraestructuras hidro-sanitarias, ya que con frecuencia no existen registros de la descripción y los cálculos realizados para las mismas, como tampoco manuales de operación para los ejecutores ni recomendaciones para los usuarios, lo que dificulta su funcionalidad a través del tiempo, sobre todo cuando se trata de tecnologías no familiares, en consecuencia, se pretende el uso de tecnologías sencillas, acompañadas de educación y capacitación permanentes que logren la apropiación tecnológica con posibilidades de adecuación in situ

\section{Referencias}

Aboites Aguilar, L. (2009). La decadencia del agua de la nación: estudio sobre desigualdad social y cambio político en México, segunda mitad del siglo XX. México, D.F: Colegio de México, Centro de Estudios Históricos.

Alvarado Granados, A. R., González Carmona, E., Díaz Cuenca, E. (2013). Los componentes del sistema del manejo del agua en una cuenca rural: aproximación metodológica. México.

Alvarado Granados, A. R., Díaz Cuenca, E., Peñuelas Guerrero, A. G. (2013). Reporte de investigación: programa de saneamiento de la cuenca del Arroyo El Muerto. Mimeo. 
Aparicio Mijares, F. J. (2006). Fundamentos de hidrología de superficie. México: Limusa.

Arias Chávez, J. (2014). Xochicalli A.C. Tecnologías inteligentes para sustentar el desarrollo. Recuperado de: http://www.xochicalli.org.mx/

Balairón Pérez, L. (2002). Gestión de recursos hídricos. Barcelona, España: Edición UPC.

CAEM Comisión del Agua del Estado de México (2001). Reglamento Interior de la Comisión del Agua del Estado de México. Periódico Oficial del Estado Libre y Soberano de México. 21 de diciembre de 2001. Toluca de Lerdo, México: Gaceta de Gobierno.

Cano Alvarán, O. L. (2004). Metodología para la concertación con los actores en la gestión de proyectos de desarrollo. Serie de Documentos: Pensamiento Administrativo Público. Escuela Superior de Administración Pública. Bogotá, Colombia: Imprenta Nacional de Colombia.

Coronado X. (2008). El análisis de sistemas como procedimiento estratégico para elaborar un modelo de evaluación institucional. Revista Entretemas. 10(5), 15-32.

Chávez Cortés, M. M. y Chávez Cortés J. M. (2009). El enfoque de estudios en la planeación de la conservación del agua. Innovación tecnológica, cultura y gestión del agua. Nuevos retos del agua en el Valle de México. (195-210). México D.F.: UAM-Porrúa.

Dávila León, O. (1996). Actores sociales y derechos ciudadanos. Revista Última Década, Centro de Estudios Sociales, Universidad de Valparaiso, Chile. (5), 1-5.

Dávila León, O. (1993). Los dilemas de la constitución de actores sociales. Revista Última Década. Centro de Estudios Sociales, Universidad de Valparaíso, Chile. (1), 1-11

Díaz Delgado, C., Esteller Alberich, M. V., López-Vera, F. (2006). Recursos Hídricos. Conceptos básicos y estudios de caso en Iberoamérica. México: CIRA, UAEMex y RIPDA.

Díez Pérez, J. A. (2012). Diagnóstico Geohidrológico del Acuífero del Valle de Toluca. Experiencias en el tratamiento de aguas residuales domésticas en el Estado de México. Toluca. México: Universidad Autónoma del Estado de México.

Dourojeanni, A. C. (2010). Redefinición de los alcances de las acciones de manejo de cuencas. Santiago de Chile: Fundación Chile. 
Dourojeanni, A. C. (2008). Los enfoques de gestión integrada de recursos hídricos y de cuencas. Wageningen UR.

Dourojeanni, A., Jouravlev, A., Chávez, G. (2002). Gestión del agua a nivel de cuencas: teoría y práctica. Recursos naturales e infraestructura. Santiago de Chile. Chile: CEPAL Naciones Unidas.

Falkenmark, M. (2003). Water management and ecosystems: living with change. Vol. 9. Estocolmo, Suecia: Global Water Partnership, Back Ground Papers.

FAO Food and Agriculture Organization of the United Nations, 2007: La nueva generación de programas y proyectos de gestión de cuencas hidrográficas. Roma, Italia: Estudio FAO, Montes.

Freitas, M. (2006) De las (¿exageradamente?) controvertidas relaciones entre la EDS y la EA hasta su contribución para lo que la década de las naciones unidas debe y puede ser. Environment and intercultural education for sustainability. Foundations and Praxis. México. Recuperado de: http://www.reseau-amerique-latine.fr/ceisal-bruxelles/ CyT-MA/CyT-MA-2-VEGA-PEDRO-\&-AL.pdf

García Charria, W. (2015). El sistema complejo de la cuenca hidrográfica. Colombia: Universidad Nacional de Colombia. Recuperado de: http://www.unalmed.edu.co/ poboyca/documentos/documentos1/documentos-Juan\%20Diego/Plnaifi_Cuencas_Pregrado/Sistema\%20CuencaHidrogr\%E1fica.pdf.

García, A. O., Galindo Sosa, J. A. (2015). Los Consejos de Cuenca y la participación social en la gestión del agua. Revista Cuencas de México (1), Año 1, abril-junio 2015, 26-31, GEM Gobierno del Estado de México (2015). Constitución Política del Estado Libre y Soberano de México. Gaceta de Gobierno. México.

GEM Gobierno del Estado de México (2011). Ley del Agua del Estado de México. Gaceta del Gobierno del Estado de México. Decreto Número 52, 2013. México. Recuperado de: http://www.edomex.gob.mx/ legistelfon/doc/pdf/gct/2011/jul223.PDF.

GEM Gobierno del Estado de México (2010). Conservación y rehabilitación de los recursos suelo ya agua de Cuenca Alta del Río Lerma Región 2 Lerma. Plan Maestro para la restauración ambiental de la Cuenca Alta del Río Lerma. México: Gobierno del Estado de México. 
GFM Gobierno Federal Mexicano (2014a). Constitución Política de los Estados Unidos Mexicanos. Constitución publicada en Diario Oficial de la Federación el 5 de febrero de 1917. Última reforma publicada DOF 07-07-2014. México, Distrito Federal: Diario Oficial de la Federación

GFM Gobierno Federal Mexicano, (2014b). Ley de Aguas Nacionales. México: Diario Oficial de la Federación.

GFM Gobierno Federal Mexicano (2014c). Ley General del Equilibrio Ecológico y la Protección al Ambiente, 1988. México, D. F.: Diario Oficial de la Federación.

INEGI Instituto Nacional de estadística e Informática (2010). Censo de Población y Vivienda. México: INEGI

Ocoyoacac, H. Ayuntamiento (2013). Plan de Desarrollo Municipal 20132015. Ocoyoacac, México.

Oré, M. T.; Geng, D. (2010). Políticas públicas del agua en el ámbito regional: Las vicisitudes en la conformación del consejo de cuecas Ica-Huancavelica. Perú: Pontificia Universidad Católica del Perú.

Pozo Solís, Antonio (2007). Mapeo de Actores Sociales. Documento de trabajo PREVAL-FIDA. Lima Perú: PREVAL-FIDA.

RAE Real Academia Española (2014) Diccionario de la lengua española, $23^{a}$ edición. España.

RIDCGIRH Red Internacional para el Desarrollo de Capacidades en la Gestión Integrada del Recurso Hídrico (2005). Planes de gestión del recurso hídrico. Manual de capacitación y guía operacional. Marzo. Cap-Net, Global Water Paternship, UNDP.

INEGI Instituto Nacional de Estadística y Geografía, Dirección General de Geografía y Medio Ambiente. (2014). Documento Técnico Descriptivo de la Red Hidrográfica 12Aa. [Hoja topográfica]. Escala 1:50,000. Edición 2.0. México: INEGI.

Manning, E. C. (1967). Political Realignment: A Challenge to Thoughtful Canadian. USA: McClelland and Stewart, Universidad de California.

Mayo, B. (2007) Los pueblos otomianos del Valle de Toluca. San Pedro Atlapulco, historia prehispánica y colonial. México D.F: Grupo Jaguar Impresiones

Savory, A. (2005). Manejo holístico. Un nuevo marco metodológico para la toma de decisiones. México, DF: SEMARNAT, INE, FMCN. 
Tapella, Esteban (2007). El mapeo de actores claves (versión preliminar). Documento de trabajo del proyecto "Efectos de la biodiversidad funcional sobre procesos ecosistémicos, servicios ecosistémicos y sustentabilidad en las Américas: un abordaje interdisciplinario". Córdoba, Argentina: Universidad Nacional de Córdoba, Inter-American Institute for Global Change Research (IAI).

UNAD Universidad Nacional Abierta y a Distancia (2015). La cuenca hidrográfica como sistema. Bogotá, Colombia. Recuperado de: http:// datateca.unad.edu.co/contenidos/201520/TGSexe/leccin_25_la_ cuenca_hidrogrfica_como_sistema.html.

Vega Marcote, P., Freitas, M., Álvarez Suárez, P., Fleuri, R. (2008). Educación Ambiental e Intercultural para un Desarrollo Sostenible: aportaciones conceptuales y metodológicas para su fundamentación. México. Recuperado de: http://www.reseau-amerique-latine. fr/ceisal-bruxelles/CyT-MA/CyT-MA-2-VEGA-PEDRO-\&-AL.pdf

Von Bertalanffy, L. (1976). Teoría general de los sistemas. México: Fondo de Cultura Económica. 